\title{
Bulk binding approach for PMIPv6 protocol to reduce handoff latency in IoT
}

\author{
Adnan J. Jabir \\ Departement of Computer Science, University of Baghdad, Iraq
}

\begin{abstract}
Mobility management protocols are very essential in the new research area of Internet of Things (IoT) as the static attributes of nodes are no longer dominant in the current environment. Proxy MIPv6 (PMIPv6) protocol is a network-based mobility management protocol, where the mobility process is relied on the network entities, named, Mobile Access Gateways (MAGs) and Local Mobility Anchor (LMA). PMIPv6 is considered as the most suitable mobility protocol for WSN as it relieves the sensor nodes from participating in the mobility signaling. However, in PMIPv6, a separate signaling is required for each mobile node $(\mathrm{MN})$ registration, which may increase the network signaling overhead and lead to increase the total handoff latency. The bulk binding approaches were used to enhance the mobility signaling for MNs which are moving together from one MAG to another by exchanging a single bulk binding update message. However, in some cases there might be several MNs move at the same time but among different MAGs. In this paper, a bulk registration scheme based on the clustered sensor PMIPv6 architecture is proposed to reduce the mobility signaling cost by creating a single bulk message for all MNs attached to the cluster. Our mathematical results show that the proposed bulk scheme enhances the PMIPv6 performance by reducing the total handoff latency.
\end{abstract}

Copyright $@ 2019$ Institute of Advanced Engineering and Science. All rights reserved.

\section{Corresponding Author:}

Adnan J. Jabir,

Departement of Computer Science, University of Baghdad, 10071, Al-Jadriya, Baghdad, Iraq. Email: adnanjjabir@gmail.com

\section{INTRODUCTION}

In recent years, the Internet of Things (IoT) is getting much attention by industry and academia due to its new trend of connecting different types of devices to the Internet without human intervention [1]. Wireless Sensor Networks (WSN) are considered as the core of IoT which are characterized by low bandwidth, power, cost, processing, and memory [2], [3]. With the advent of the IoT and ubiquitous computing, the need has emerged to design protocols for connecting WSN to Internet. Since Internet is the most widespread network, connecting the WSN to it in order to disseminate the sensed data is essential for making the IoT into a reality [4]. Integrating the Internet Protocol (IP) with WSN would indeed facilitate WSN to interconnect with other IP networks and to capitalize the existing Internet infrastructure and IPapplications to sensor networks [5], [6].

In addition, mobility has also an important role in realizing the IoT by achieving "anywhere, anytime" communication. Mobility management protocols are very essential in the new research area of IoT, it aims to track and locate the Mobile Nodes (MNs) efficiently to provide the user with full access to information irrespective of their locations.

Host based mobility management protocols including MIPv6 [7], FMIPv6 [8], and HMIPv6 [9], involve the MNs in the mobility process and generally introduce a significant overhead on the MNs which 
should install complex mobility protocols. In addition, in case where an MN has no capability to transmit the mobility related signaling, host-centric mobility management protocols will be no longer functional [10]. Therefore, methods for relieving MNs from participating in mobility process and reducing handoff latency, packet loss, and communication path are indeed essential for the IoT [11].

Proxy Mobile IPv6 (PMIPv6) [12] was standardized by the IETF NETLMM working group as a network-based mobility management protocol to solve these problems associated to the host-based protocols by relocating the mobility procedure from MNs to the network components. PMIPv6 adds two functional entities which are the Mobile Access Gateway (MAG) and the Local Mobility Anchor (LMA). The main role of LMA is to maintain reachability to the MN address while it moves in the local PMIPv6 domain. The MAG is responsible for detecting the $\mathrm{MN}$ movements and initiating the required signaling to register the $\mathrm{MN}$ with the LMA.

The salient feature of the PMIPv6 is its network-based attribute, in which, the network detects the node mobility and initiates the required mobility signals, so that, it relieves the MN from participating in the handoff process. This feature allows MNs which installed only the conventional IP protocol to roam freely between wireless networks without any need for the installation of complicated mobility management protocols [13]. Considering both the network-based feature of PMIPv6 and the low capabilities of WSN which makes the use of host-based mobility protocols like MIPv6 insufficient; researchers are motivated to use PMIPv6 for managing the WSN mobility. For example, the SPMIPv6 was proposed by Islam in [14] as an adaptation of the PMIPv6 protocol.

Although PMIPv6 fits with the WSN needs as it reduces the signaling cost, mobility cost, and the level of energy consumption. However, in PMIPv6, for each node a separate handoff signaling should be exchanged between MAG and LMA to register the MN's new location. This may cause an extensive network signaling if there is a large number of moving MNs [15].

Several research works were done to reduce the signaling overhead by introducing the bulk registration principles. By using the bulk registration, a single binding message is required to register a number of MNs which are moving together from one MAG to another. However, MNs are not always moving together between two MAGs, they may move at the same time among different MAGs. These MNs do not share any common property that allows the conventional bulk schemes to register them by a single bulk message. In this paper, we propose a new bulk registration scheme based on the Clustered Sensor PMIPv6 (CSPMIPv6) [16] architecture, named BCSPMIPv6, to address the problem of MNs registrations which are moving at the same time but between different MAGs. Although MNs move among different MAGs, a group of these MNs belong to the same cluster. Thus, the clustering principle provides the required common property for those MNs in order to create a single bulk registration message.

\section{RELATED WORK}

Reducing the handoff signaling cost for the MNs which are moving together was addressed by several researches. The mobile node group identifier was first introduced in [17] to identify a set of mobile nodes which have common characteristics. It provides performing the required binding update function for a set of MNs using only a single binding message rather than using multiple atomic binding per mobile node. The mobile node group identifier is exchanged between LMA and MAGs and can be used to optimize the reregistration or revocation functions for a set of MNs. The bulk re-registration allows MAGs to extend the binding life time for a set of MNs belong to the same group by requesting a bulk re-registration message. In this case, the re-registration messages are independent on the number of MNs; instead it depends on the number of MAGs [18].

To optimize the bulk message size, authors in [15] proposed a new optimized bulk message format based on the bitmap structure. In the conventional bulk registration, the message is built by simply concatenating multiple PBU messages tied for the MNs which are belonging to the same group. In this case, the mobility options are repeated for each MN which enlarges the bulk message size. Instead, by using the bitmap structure, only a single instance of the mobility options is added to the bitmap matrix. Hence, the bulk message size is reduced and there will be no need for breaking the bulk message into multiple parts to avoid the packet drop.

Reducing the number of binding update messages for a numerous number of MNs moving together has attracted researchers to apply the bulk registration principles into the network mobility domains. Authors in [19] introduced a bulk binding update to the intelligent transportation systems where a number of mobile routers are moving between access networks. By assigning a mobile node group identifier to this set of moving routers, the overall signaling overhead required during the periodic binding update is reduced.

When mobile router moves between MAGs in the LMA domain, a large number of binding update and binding acknowledgement messages is required to be exchanged for MNs registration or update their 
new locations. Since these MNs are moving together as a single unit, authors in [20], [21] introduced the use of bulk registration to combine individual binding messages of all nodes in mobile network and sends them together as a single binding message. Heading this way, handoff time and number of signaling messages to be exchanged during mobile network movement are reduced.

The conventional bulk scheme solves the group mobility scenarios, where a group of MNs move between two MAGs, by introducing the bulk PBU and PBA messages to register a group of MNs using a single bulk message. In many scenarios, as shown in Figure 1, MNs may move individually among different MAGs, but they move at the same time. However, the current solutions are not able to use the bulk registration for such mobility scenario since there is no common characteristic shared by these MNs. This is due to the distribution of MNs among different MAGs which makes it difficult to create a single bulk message for these MNs. Therefore, the case when a number of MNs belonging to different MAGs, move at the same time to other MAGs need to be addressed to enhance the PMIPv6 performance.

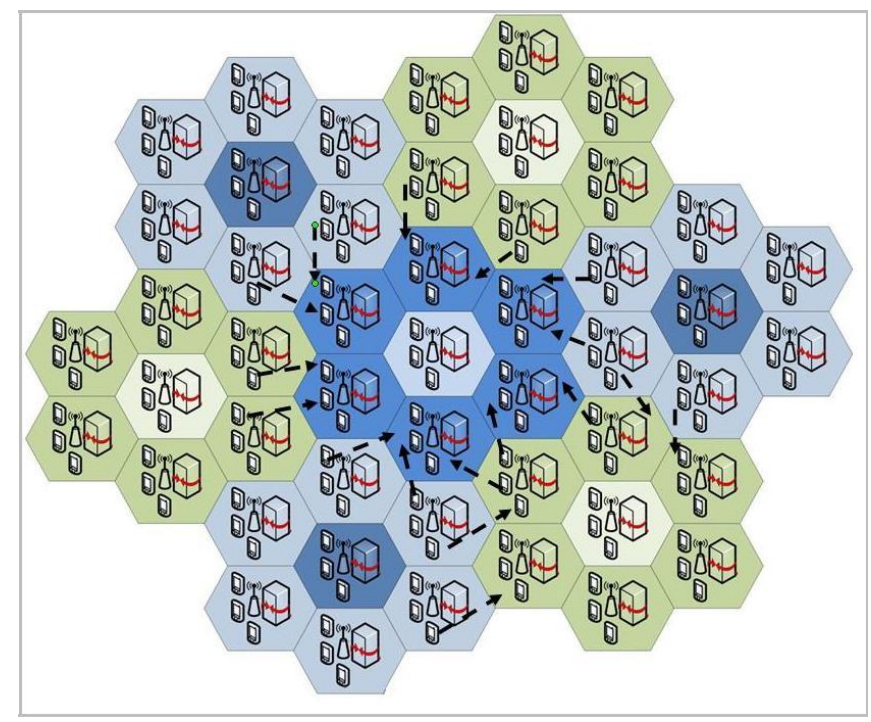

Figure 1. Node mobility scenarios

\section{PROPOSED SCHEME}

In this section, the CSPMIPv6 architecture with it signaling and control messages are presented first, and then we deliberate on the proposed bulk registration scheme.

\subsection{CSPMIPv6 architecture}

The main idea behind the CSPMIPv6 is to divide the PMIPv6 domain into a number of local subdomains. Each sub-domain is represented as a cluster which constitutes a group of MAGs with a one Head MAG (HMAG). The HMAG is one of the LMA's domain MAGs which is elected to play the role of a cluster head [16].

The main roles of the HMAG are: providing a local mobility management for each cluster, reducing the handoff latency for the MNs moving inside the cluster, and is used to provide a sufficient buffering scheme for the MNs during the handoff process [16].

In CSPMIPv6, a number of messages should be exchanged among entities to perform the registration and handoff processes. These messages include the Proxy Binding Update (PBU), Proxy Binding Acknowledge (PBA), Local PBU (LPBU), Local PBA (LPBA). In addition, all the LMA, HMAGs, and the MAGs maintain their own binding list to store the required information of the MN's current location [16]. The PBU and PBA are used to register the MN with the LMA in the same manner of the [12], while the LPBU and the LPBA are used between MAGs and their HMAGs for the local MN's registration.

When MN enters the CSPMIPv6 domain for the first time, the registration of the MN's information in the MAG, HMAG, and the LMA is required, as follows: once a MAG detects an attachment of an MN, it sends an LPBU request to its HMAG contains the MN identifier (MN-ID). Upon receiving the LPBU by HMAG, it searches its BUL for an MN entry. Since it is a new MN registration process, the HMAG will not find a matching MN entry. Therefore, the HMAG sends a PBU request to the LMA containing the MN's 
identifier and the requesting HMAG. Upon receiving the PBU, the LMA creates a new BCE for the MN and sends a PBA message back to the requesting HMAG. Then the LMA sets up a global bidirectional tunnel with the corresponding HMAG to be used for routing the traffic to and from the MN. Upon receiving the PBA message, the HMAG registers the MN in its BUL and sends an LPBA message back to the corresponding MAG containing the MN prefix. Then the HMAG configures a local tunnel with the MAG to be used for routing the packets destined to the MN. Upon receiving the LPBA by the requesting MAG, it registers the $\mathrm{MN}$ in its BUL and sends a router advertisement message to the requesting node.

\subsection{Bulk CSPMIPv6 scheme}

Conventional bulk schemes assume that MNs move as a group from one or more MAGS to one destination MAG. As it can be seen from Figure 2, when a MAG detects the attachment of a group of MNs, it creates one bulk PBU message to register these MNs with LMA [15]. However, if a number of MNs move at the same time among different MAGs such that a single MN moves to one MAG, the LMA receives a number of PBU equals to the number of the attached MNs, as shown in Figure 3. Thus, we propose a bulk scheme which can cope with this case by creating a single bulk message for MNs move among different MAGs.

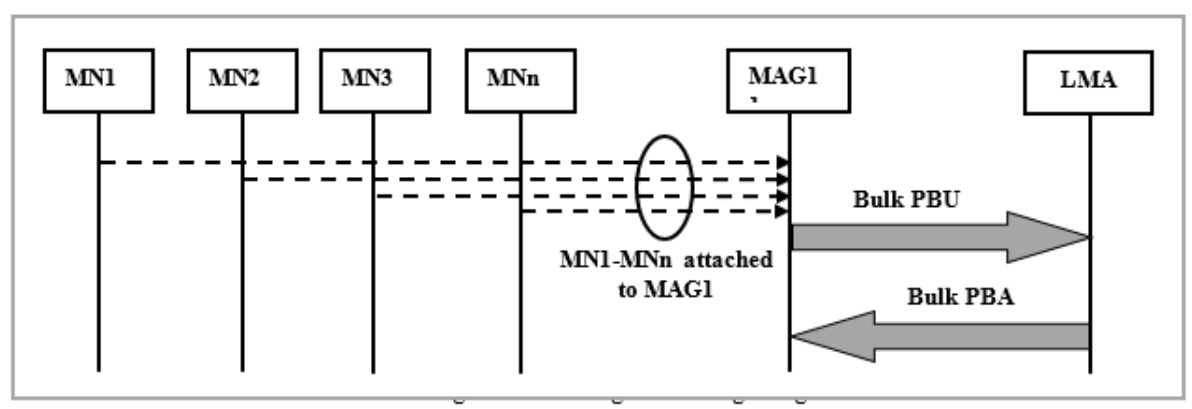

Figure 2. Bulk Registration Signaling

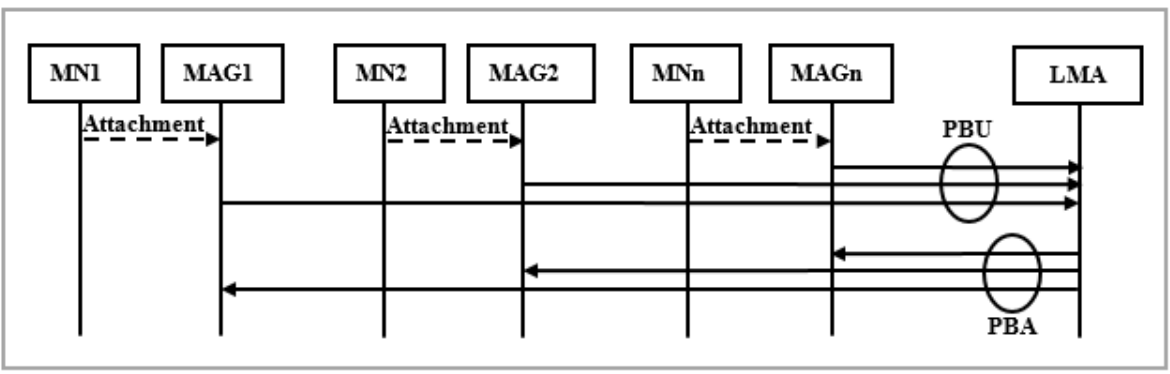

Figure 3. Single Registration per MN

The proposed scheme is designed based on the CSPMIPv6 architecture to reduce the extensive network signaling by using a single bulk binding message for every group of MNs which have a common property. By introducing the bulk registration to the CSPMIPv6 architecture, the case when MNs move individually among different MAGs can be solved by utilizing the HMAG entity. As shown in Figure 4, when MNs move among different MAGs, each MAG sends a single LPBU message to the HMAG which in turn collects all these requests and creates a single bulk PBU message to the LMA.

In the proposed scheme, when a group of MNs move between two MAGs belonging to one or more clusters, the new MAG sends a single bulk LPBU to its HMAG for the MNs group registration. If the source and destination MAGs belong to the same cluster, the HMAG replies by a single bulk PBA message for this group registration. Adversely, if the MAGs belong to different clusters, the HMAG sends a bulk PBU to the LMA. On the other hand, when MNs move between MAGs belonging to different clusters, each MAG sends a normal or bulk LPBU to the HMAG which in turn sends a bulk PBU to the LMA for these MNs 
registrations. The HMAG collects the PBU requests from its attached MAGs and sends it as a single bulk PBU message to the LMA.

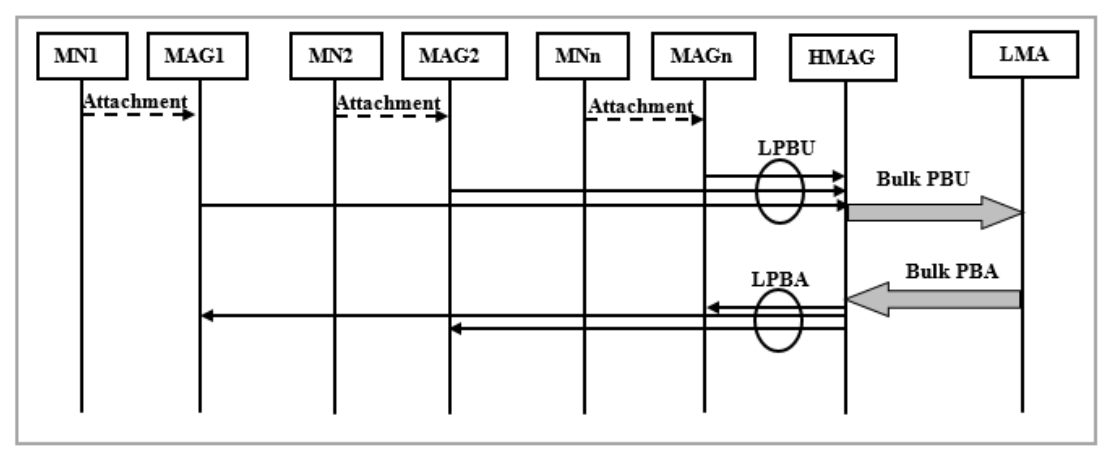

Figure 4. Proposed Bcspmipv6 Bulk Scheme Signaling

\section{PERFORMANCE EVALUATION}

In this section, the performance of the proposed BCSPMIPv6 is evaluated and compared against the basic PMIP and the conventional bulk schemes (BPMIPv6), in terms of total handoff latency.

\subsection{Analytical model}

In this section, the total Handoff Latency (HL) is derived to compare the performance of the proposed BCSPMIPv6 with the basic PMIPv6 and BPMIPv6 schemes. It is necessary to mention here that we focus on the intra-domain mobility only (i.e. the MN moves within the same LMA domain). In addition, for the proposed BCSPMIPv6 scheme we consider both the intra- and inter-cluster mobility within the same domain. The performance notations used in the analysis, are presented in Table 1.

Table 1: Parameters for Performance Analysis

\begin{tabular}{ll}
\hline \multicolumn{1}{c}{ Parameter } & \multicolumn{1}{c}{ Description } \\
\hline $\mathrm{C}_{\mathrm{x}-\mathrm{y}}$ & Hop count between nodes $\mathrm{x}$ and $\mathrm{y}$ \\
$\mathrm{S}_{\text {ctrl }}$ & Size of a control packet (byte) \\
$S_{B u l k}^{i}$ & Size of bulk message for MAGi or $\mathrm{HMAG}_{\mathrm{i}}$ \\
$I P_{h s}$ & IPv6 packet header size \\
$M N c_{i}$ & Number if MNs in MAG $\mathrm{MMAG}_{\mathrm{i}}$ \\
$t$ & Unit transmission cost of packet per a wired link \\
$B w$ & Link bandwidth \\
$H L_{x}$ & Handoff Latency for $x$ protocol \\
\hline
\end{tabular}

\subsection{Signaling cost analysis}

In this section, the MN's handoff latency is derived for all schemes under consideration. We assume the distance between any two entities is symmetric. In addition to simplify the mathematical calculations, we are not going to consider the AAA functions and the processing cost for LMA, MAGs, HMAGs, and the intermediate routers.

\subsubsection{Basic PMIPv6}

In the basic PMIPv6 protocol, the location update is performed by exchanging the PBU and PBA messages between the LMA and MAGs when the MN moves from one MAG to another, which can be derived as follows:

$$
H L_{P M I P}=\frac{S_{c t r l}}{\mathrm{Bw}} \times 2 \mathrm{t} \times \mathrm{C}_{\mathrm{MAG}-\mathrm{LMA}} \times \mathrm{MNC}
$$

\subsubsection{BPMIPv6}

In BPMIPv6 scheme, the bulk PBU and PBA messages are exchanged between MAGs and LMA to register the MNs which are moving as a group between MAGs. Therefore, the handoff latency for BPMIPv6 
can be derived as follows:

$$
\begin{aligned}
& H L_{B P M I P}=\sum_{\mathrm{i}=1}^{\mathrm{MAgs}} S_{B u l k}^{i} \times 2 t \times C_{L M A-M A G} \\
& S_{\text {Bulk }}^{i}=M N c_{i} \times\left(S_{c t r l}-\mathrm{IP}_{\mathrm{hs}}\right)+\mathrm{IP}_{\mathrm{hs}}
\end{aligned}
$$

\subsubsection{BCSPMIPv6}

In BCSPMIPv6, the intra-cluster mobility is controlled by HMAG through exchanging the LPBU/LPBA messages with its attached MAGs. On the other hand, the inter-cluster mobility requires the involvement of LMA to update the MN's information using the PBU and PBA messages. Accordingly, the total handoff latency, which is the sum of intra-cluster handoff and inter-cluster handoff signaling costs, can be derived as follows:

$$
H L_{B C S P M I P}=\sum_{i=1}^{H M A G S} S_{B u l k}^{i} \times 2 t \times C_{H M A G-L M A}+\sum_{j=1}^{M A G S} S_{B u l k}^{j} \times 2 t \times C_{M A G-H M A G}
$$

\section{NUMERICAL RESULTS}

The numerical results based on the derivations in the previous section are presented here. The assumptions and parameter values used in this research are shown in Table 2. In addition, a simulator has been designed to simulate the MN mobility based on fluid-flow mobility model, in which, MNs are rarely changing their speed and directions. The number of MNs crossing MAGs at every simulation time is obtained by the simulator.

Table 2: Parameters Values

\begin{tabular}{cc}
\hline Parameter & Default Value \\
\hline$t$ & 2 \\
$v$ & $25(\mathrm{~m} / \mathrm{s})$ \\
$R$ & $500(\mathrm{~m})$ \\
$\mathrm{S}_{\text {ctrl }}$ & 96 bytes \\
$I P_{h s}$ & 40 bytes \\
$C_{M A G-L M A}$ & 16 \\
$C_{M A G-M A G}$ & $\sqrt{\mathrm{MAGs}}$ \\
MNc & 1000 \\
Mobility model & Fluid-flow \\
\hline
\end{tabular}

Figure 5 shows the effect of the wired link delay on the handoff latency. It can be clearly seen that the handoff latency of all schemes is increased with the increment of the wired link delay. However, BCSPMIPv6 performs better than other schemes because of the reduction of the signaling required for performing the inter- and intra-cluster mobility.

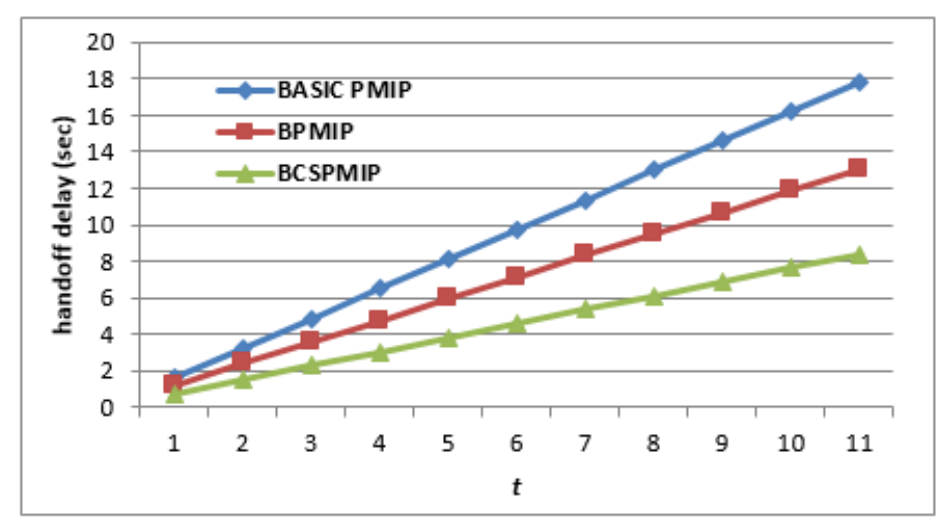

Figure 5. Impact of Wired Link Delay on the Handoff Latency

The handoff latency is proportional to the number of hopes between the communicating entities. Figure 6 shows the impact of LMA-MAG distance on the handoff latency. The basic PMIPv6 incurs the 
highest handoff latency due to its highly dependent on the LMA. The low effect of the distance on the BCSPMIPv6 is due to the use of bulk registration for the inter-cluster mobility and the exclusion of LMA from participating in the intra-cluster operations.

The handoff latency is also affected by the number of MNs moving between MAGs, which is shown in Figure 7. It can be seen that when the number of MNs increased, the handoff delay is increased for all schemes. However, BCSPMIPv6 presents the lowest handoff delay due to rely the intra-cluster mobility on the HMAGs and the use of bulk registration for the inter-cluster mobility.

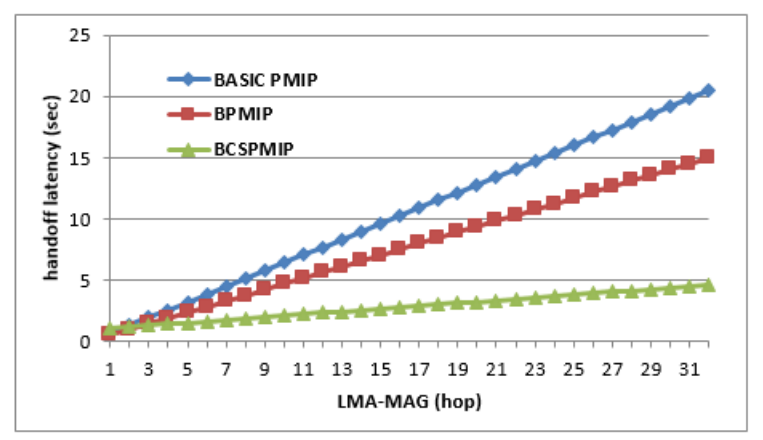

Figure 6. Impact of LMA-MAG Distance on the Handoff Latency

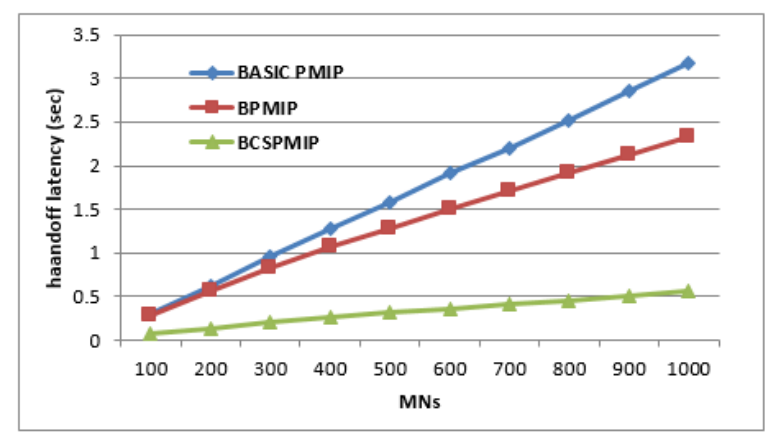

Figure 7. Impact of the Number of MNs on the Handoff Latency

\section{CONCLUSIONS}

In this paper, the BCSPMIPv6 is proposed as a new bulk scheme based on the CSPMIPv6 architecture to reduce the total handoff latency. The main idea of the clustered PMIPv6, which is the base of BCSPMIPv6, is to divide the proxy domain into sub-domains formed as clusters, such that the intra-cluster handoff is performed locally by the HMAG which thereby leads to low handoff latency. In our proposed BCSPMIPv6 scheme, the MNs which are moving among different MAGs at the same time are registered by using a single bulk PBU message. Our analysis and numerical results prove that the proposed BCSPMIPv6 scheme outperforms the previous work including the basic PMIPv6 and the conventional bulk scheme BPMIPv6 in terms of handoff latency. Future investigations could focus on the implementation of the BCSPMIPv6 scheme to validate its scalability using a high and random mobility environment.

\section{REFERENCES}

[1] Jamali, M.S., P. Kumar, and U.A. Khan, "Internet of Things: Architecture \& Integration with Other Networks," First International Conference on Modern Communication \& Computing Technologies (MCCT'14), 2014.

[2] Akyildiz, I.F., et al., "Wireless sensor networks: a survey," Computer networks, vol. 38(4), pp. 393-422, 2002.

[3] Mahyastuty, Veronica Windha, and M. Sigit Arifianto, "Wireless Sensor Network over High Altitude Platform." Telecommunication, Computing, Electronics and Control (TELKOMNIKA), vol. 16(1), pp. 129-133, 2018.

[4] Rodrigues J.J. and P.A. Neves, "A survey on IP-based wireless sensor network solutions," International Journal of Communication Systems, 23(8), pp. 963-981, 2010. 
[5] Zinonos Z. and V. Vassiliou, "Inter-mobility support in controlled 6LoWPAN networks," in GLOBECOM Workshops (GC Wkshps). 2010: IEEE.

[6] Chen Y.-S., C.-S. Hsu, and H.-K. Lee, "An enhanced group mobility protocol for 6LoWPAN-based wireless body area networks," In Wireless Communications and Networking Conference (WCNC). 2013: IEEE.

[7] Johnson D., C. Perkins, and J. Arkko, "Mobility support in IPv6," IETF RFC 3775, 2004.

[8] Soliman H., L. Bellier, and K.E. Malki, "Hierarchical mobile IPv6 mobility management (HMIPv6),", IETF RFC 4140, 2005.

[9] Koodli, R., "Fast handovers for mobile" IPv6, IETF RFC 4068, 2005.

[10] Jabir A.J., Shamala S., Zuriati Z. and Hamid N.," A comprehensive survey of the current trends and extensions for the proxy mobile IPv6 protocol," IEEE Systems Journal, 2015.

[11] Bojkovic Z., B. Bakmaz, and M. Bakmaz, "Some Challenging Issues for Internet of Things Realization," Proceedings of the 12th International Conference on Data Networks, Communications, Computers (DNCOCO '13), March 2013.

[12] Gundavelli S., et al., "Proxy mobile ipv6," IETF RFC 5213, 2008.

[13] Hashim, Aisha, Azana Hafizah Mohd Aman, and Huda Adibah Mohd Ramli. "Throughput and Handover Latency Evaluation for Multicast Proxy Mobile IPV6," Bulletin of Electrical Engineering and Informatics (BEEI), vol. 6(4), pp. 311-316, 2017.

[14] Islam M.M. and E.-N. Huh, "Sensor proxy mobile IPv6 (SPMIPv6)—A novel scheme for mobility supported IPWSNs," Sensors, vol 11(2), pp. 1865-1887, 2011.

[15] Jeyatharan M. and C.-W. Ng, "Bulk PBU using Bitmaps", 2009.

[16] Jabir, A.J., et al., "A cluster-based proxy mobile IPv6 for IP-WSNs," EURASIP Journal on Wireless Communications and Networking, pp. 1-17, 2012.

[17] Gundavelli S., et al., "Mobile Node Group Identifier option," 2009.

[18] Krishnan S., et al., "Bulk Re-registration for Proxy Mobile IPv6," 2009.

[19] Vasu K., S. Mahapatra and C. Kumar, "Bulk Binding Update Procedure for Pmipv6 Based Intelligent Tansportation Systems" 2012.

[20] Jebaseeli S.A. and J. Sundararajan, "Reducing Binding update in NEMO Supported PMIPV6," International Journal of Computer Trends and Technology (IJCTT), vol. 6(3), pp.150-157, 2013.

[21] Guan, Jianfeng, Ilsun You, Changqiao Xu, and Hongke Zhang, "The PMIPv6-Based Group Binding Update for IoT Devices," Mobile Information Systems, 2016.

\section{BIOGRAPHY OF AUTHORS}

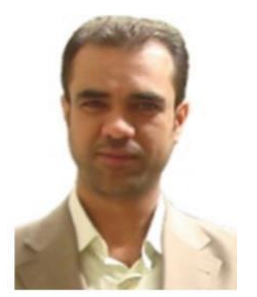

Adnan J. Jabir received the B.Sc. and M.Sc. degrees in Computer Science from the University of Baghdad, Iraq in 1995 and 1998, respectively. He received his Ph.D. degree at the Department of Communication Technology and Networks, Faculty of Computer Science and Information Technology, University Putra Malaysia (UPM), Malaysia in 2015. He is currently a senior lecturer at University of Baghdad, college of Science, computer Science Department. His major research interests are wireless sensor networks, ubiquitous computing, and network mobility management. 